\title{
Designing User Interfaces from Analyses of Users' Tasks
}

\author{
Peter Johnson, Stephanie Wilson and Hilary Johnson \\ HCI Laboratory, Department of Computer Science, \\ Queen Mary \& Westfield College, University of London, \\ Mile End Road, London E1 4NS, U.K. \\ pete, steph, hilaryj@dcs.qmw.ac.uk
}

\begin{abstract}
This tutorial provides a detailed introduction to task analysis and task-based design. The focus of task analysis is the description of users' work tasks, while the focus of task-based design is designing from the perspective of users' work. Techniques from psychology, ethnomethodology and sociology are used to analyse and describe users' work tasks. A framework for modelling work tasks (Task Knowledge Structures) is used to represent relevant task information. Guidelines are provided to help envision how current tasks might be changed and improved through the design of interactive systems. Descriptions of envisioned tasks form the basis of interaction design, thus ensuring that designs are focused on the tasks they are intended to support.
\end{abstract}

KEYWORDS task analysis, task-based design, work-tasks, design guidelines, envisioning design

\section{INTRODUCTION}

Task analysis is concerned with understanding work. It is an important $\mathrm{HCI}$ technique that enables users to be involved in the design process and enables designers to understand users' work. Task-based design is concerned with developing interactive systems from the perspective of the users' work and usage of the designs. Taskbased design provides work-tasks as the basis for the envisionment of new work and the design of new systems.

Task analysis can be used at multiple points in the design process, to identify and scope the situation of concern, to provide a well-grounded and detailed description of the current work situation, to identify scenarios of usage, to envision new ways of working and to provide benchmark tasks against which designs can be evaluated. Moreover, task analysis can be carried out at various levels of detail, ranging from gaining an overview of the range and type of tasks in a given organisation to providing a detailed description of how a complex activity is carried out. The aim of this tutorial is to provide $\mathrm{HCI}$ professionals with appropriate knowledge of how to carry out task analysis, how to envision potential changes to work tasks and how to design systems from the perspective of these tasks.

\section{OBJECTIVES AND MOTIVATION}

This is an intermediate level tutorial intended for $\mathrm{HCI}$ professionals including designers and developers of user interfaces, human factors practitioners and researchers. The overall objective of the tutorial is to teach the benefits and use of task analysis and task-based user interface design. There are three main aspects to this:

- To introduce the ideas of task analysis and task-based user interface design, and to consider some of the advantages and limitations of such approaches.

- To describe a selection of methods and guidelines for analysing tasks, modelling tasks and developing user interface designs.

- To provide practical experience in the application of these techniques.

A major motivation for the tutorial stems from recent studies of design practice which indicate poor take-up of task analysis and other $\mathrm{HCI}$ techniques, for reasons such as perceived cost and lack of expertise. With respect to 
cost, it can be cost-effective to introduce $\mathrm{HCI}$ techniques such as task analysis and prototyping into the design process e.g. (Mantei and Teorey, 1988). Moreover, (Rossen et al, 1988) identified that designers consider obtaining information about users and tasks as a major contributor to the generation of design ideas. In surveying design practice, (Johnson and Johnson, 1989) found that designers believed task analysis could contribute significantly to interactive system design and the resulting usability of the designed systems. Knowledge about how users perform tasks enables designers to reason about what aspects of task should or should not be supported, how they could be supported and what changes to the task will come about as a result of a new design, i.e. an envisioned task will enable designers and users to consider and reason about how the user will interact with the new design.

\section{CONTENT}

This tutorial introduces participants to the concepts and methods of task analysis and task-based design. This includes understanding the TKS approach to task analysis and task modelling (Johnson and Johnson, 1991), and the ADEPT approach to task-based design (Johnson et al, 1995), (Wilson and Johnson, 1996). Participants are provided with practical experience of conducting a task analysis and producing a task model, and then using this as the basis for designing a user interface.

The tutorial lasts one full day and involves interactive presentations, demonstrations and small group exercises. The interactive presentations introduce the concepts and approaches of task-based user interface design. The demonstrations include video and real demonstrations of task-based design. The group work focuses on taking the participants through a task analysis and developing a user interface design. This involves participants constructing the various task and interaction models, and using design guidelines to develop a user interface. One design problem is used as a running example throughout the tutorial: designing a system to support querying of airline flight information and making a booking.

\section{Introduction}

The tutorial starts by providing participants with an overview of task-based design. The aims and motivations of task-based design and some of its advantages and limitations in relation to other design approaches are considered. A demonstration is given of a complete task- based design process, from the analysis of tasks to the design of a user interface.

Task analysis and task modelling

This part of the tutorial teaches participants the concepts of task modelling and the methods of task analysis and data collection. It introduces the Task Knowledge Structures framework for modelling and analysing tasks. Practical experience is given in choosing and using one or more methods to analyse the flight booking task.

Task-based design - envisioning future work tasks

In the third part of the tutorial, the students are taught principles and guidelines for envisioning and reasoning about changes to be made to current work tasks. Group exercises again give participants the opportunity to practice these techniques.

Task-based design - developing user interface designs

The final part of the tutorial provides students with methods and guidelines for developing a user interface design from an envisioned task model, and gives them practical experience in applying these to develop a prototype design for a user interface to the flight booking system. The tutorial closes with a plenary discussion and feedback session.

\section{REFERENCES}

Johnson, H. and Johnson, P. (1989) Integrating task analysis into system design: Surveying designers' needs, Ergonomics, 32(11), 1451-1467.

Johnson, H. and Johnson, P. (1991) Task knowledge structures: Psychological basis and integration into system design, Acta Psychologica, 78, 3-26..

Johnson, P. Johnson, H. and Wilson, S. (1995) Rapid prototyping of user interfaces driven by task models, in Scenario Based Design, (ed. J.M. Carroll), Wiley.

Mantei, M.M. and Teorey, T.J. (1988) Cost/benefit analysis for incorporating human factors in the software lifecycle. Communications of the ACM, 31, 428443.

Rosson, M.B., Maass, S. and Kellogg, W.A. (1988) The designer as user: Building requirements for design tools from design practice. Communications of the ACM, 31, 1288-1298.

Wilson, S. and Johnson, P. (1996) Bridging the generation gap: From work tasks to user interface design, in Computer-Aided Design of User Interfaces, (ed. J. Vanderdonckt) Presses Universitaires de Namur, 44-55. 